\title{
Temperature effect on the lasing from a dye-doped two-dimensional hexagonal photonic crystal made of holographic polymer-dispersed liquid crystals
}

\author{
D. Luo, ${ }^{1}$ X. W. Sun, ${ }^{1}$ H. T. Dai, ${ }^{1}$ H. V. Demir, ${ }^{2,3}$ H. Z. Yang, ${ }^{4}$ and W. Ji ${ }^{4}$ \\ ${ }^{1}$ School of Electrical and Electronic Engineering, Nanyang Technological University, Nanyang Avenue, \\ Singapore 639798 \\ ${ }^{2}$ School of Electrical and Electronic Engineering and School of Physical and Mathematical Sciences, \\ Nanyang Technological University, Nanyang Avenue, Singapore 639798 \\ ${ }^{3}$ Department of Electrical and Electronics Engineering and Department of Physics, Bilkent University, \\ Balkans, Ankara 06800, Turkey \\ ${ }^{4}$ Department of Physics, National University of Singapore, 2 Science Drive 3, Singapore 117542
}

(Received 20 May 2010; accepted 25 May 2010; published online 12 July 2010)

\begin{abstract}
Temperature dependent lasing was demonstrated in a dye-doped two-dimensional hexagonal photonic crystal made of holographic polymer-dispersed liquid crystals (LCs) along $\Gamma \mathrm{M}$ direction in TE polarization. A redshift in lasing peaks was observed as the temperature increased from 25 to $45{ }^{\circ} \mathrm{C}$. The downward movement of photonic band of TE polarization, majorly caused by the decrease in the anisotropy of LC droplets with the increase in temperature, is responsible for the redshift in lasing peaks. (C) 2010 American Institute of Physics. [doi:10.1063/1.3456991]
\end{abstract}

\section{INTRODUCTION}

Polymer/liquid crystal (LC) composite is one kind of promising materials to fabricate electro-optical elements with electrically switchable/tunable properties, providing simple fabrication procedure and low cost. Polymerdispersed LCs (PDLCs) and holographic PDLCs (H-PDLCs) are representative polymer/LC composites, which have been both researched extensively in a variety of applications. ${ }^{1}$ Two-dimensional (2D) and three-dimensional (3D) photonic structures $^{2,3}$ can be sculpted into the polymer/LC composite conveniently by two-beams or multibeams interference. Recently, H-PDLC-based lasing has also been investigated in one-dimensional (1D) dye-doped gratings, ${ }^{4-6}$ where the underlying mechanism is much similar to conventional 1D distributed feedback lasers (Ref. 7) and 2D dye-doped photonic crystal (PC) ${ }^{8,9}$ In dye-doped H-PDLC PCs, the groupvelocity anomaly, ${ }^{10}$ where the group velocity is small over a wide range of wave vectors, is responsible for the possible local field enhancement.

The tunable properties of lasing based on dye-doped H-PDLC have been discussed in the presence of electric field, such as in reflection grating, ${ }^{11,12}$ transmission grating, ${ }^{5,13}$ and $2 \mathrm{D}$ square PC. ${ }^{8}$ The temperature dependent tunable properties of lasing have also been studied in PDLC (Ref. 14) and H-PDLC transmission grating, ${ }^{15}$ however, rarely in 2D H-PDLC PCs. In this paper, we shall systematically study the temperature dependence of lasing from $2 \mathrm{D}$ H-PDLC PC with hexagonal lattice structure along $\Gamma \mathrm{M}$ direction. We report a redshift in lasing peaks with the increase in temperature, which can be explained by the downward movement of photonic band, which is primarily due to the decrease in LC droplets' anisotropy with the increasing ambient temperature.

\section{EXPERIMENT}

In our experiment, the 2D H-PDLC PC with hexagonal lattice structure was holographically fabricated through the three-beam interference based on a single prism impinging by collimated $\mathrm{Ar}^{+}$laser beam $(514.5 \mathrm{~nm})$. During the polymerization process, monomers start to photopolymerize in high intensity regions while LCs diffuse into low intensity region, thus forming the columnar polymer and LC droplets after the phase separation. The exposure intensity of each beam was $15 \mathrm{~mW} / \mathrm{cm}^{2}$, with an exposure time of $120 \mathrm{~s}$. The recording area of the fabricated 2D PC sample was about 5 $\times 5 \mathrm{~mm}^{2}$ in $x-y$ plane.

The LC/prepolymer mixture syrup used to fabricate the dye-doped 2D PC with hexagonal lattice structure consisted of $63.76 \mathrm{wt} \%$ monomer, trimethylolpropane triacrylate, 7.05 wt $\%$ cross-linking monomer, $N$-vinylpyrrollidone, 0.49 wt $\%$ photoinitiator, rose bengal, 0.98 wt $\%$ coinitiator, $\mathrm{N}$-phenylglycine, 9.30 wt \% surfactant, octanoic acid, and 1.18 wt \% lasing dye, 4-dicyanomethylene-2-methyl-6-pdimethylaminostyryl-4H-pyran, all from Sigma-Aldrich, and 17.24 wt $\%$ LC, E7 $\left(n_{\mathrm{o}}=1.5216\right.$ and $\left.n_{\mathrm{e}}=1.7462\right)$, from Merck. The mixture was sandwiched in a cell, which was formed by two pieces of indium tin oxide coated glass. The cell gap was $7 \mu \mathrm{m}$.

To generate lasing from the 2D H-PDLC sample, a Q-switched frequency-doubled Nd:yttrium-aluminum-garnet pulsed laser operating at $532 \mathrm{~nm}$, with a pulse duration of 7 ns and a repetition rate of $10 \mathrm{~Hz}$, was used. A linearly polarized pumping laser, focused by a cylinder lens, was impinged on the surface of the sample along the $z$ direction in TE polarization (electric field in $x-y$ plane). A fiber coupled spectrometer along $x$ axis with a resolution of $0.6 \mathrm{~nm}$ was used to collect output lasing beams, and the output laser was measured in TE polarization (with polarization along $y$ axis). The schematic optical setup is shown in Fig. 1, and the inset 


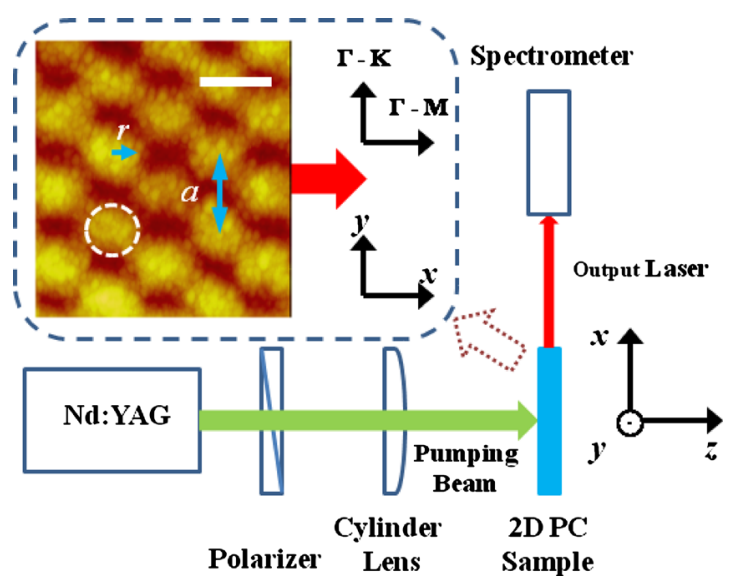

FIG. 1. (Color online) Schematic optical setup of the lasing experiment. Inset figure is an AFM image for the surface morphology of a 2D hexagonal H-PDLC PC. Scale bar: $500 \mathrm{~nm}$.

figure shows the surface morphology of the 2D H-PDLC PC with a hexagonal lattice structure imaged by an atomic force microscope (AFM). The lattice constant of the 2D PC sample, $a$, was $\sim 517 \mathrm{~nm}$, and the polymer columns have a radius $r$ of $\sim 155 \mathrm{~nm}$. The details of fabrication setup can be found elsewhere. ${ }^{9}$

\section{RESULTS AND DISCUSSIONS}

Figures 2(a)-2(c) show the lasing spectra measured along $\Gamma \mathrm{M}$ direction at temperatures of 25,35 , and $45{ }^{\circ} \mathrm{C}$, under different pumping energies of 260, 300, and $380 \mu \mathrm{J} /$ pulse. The corresponding lasing peaks, which increase with the increasing pumping energy, are $610 / 615 \mathrm{~nm}$, 620/626 nm, and 624/631 nm in Figs. 2(a)-2(c), respectively. Those lasing peaks were induced by the group-velocity anomalies, which is peculiar to 2D and 3D PCs and even exists in PCs based on H-PDLC materials with rather small index contrast. The simulation works reported by Sakoda indicated that two or more lasing peaks can be generated simultaneously in the frequency range of group-velocity anomalies of 2D PC with small index contrast, ${ }^{10}$ which gives a good explanation of our experimental spectra with two lasing peaks here. Additionally, although allowed in theory, these completing lasing peaks are preferred to appear under a higher pumping energy, due to the spatial hole burning effect. ${ }^{16}$ From Figs. 2(a)-2(c), we can find that when the
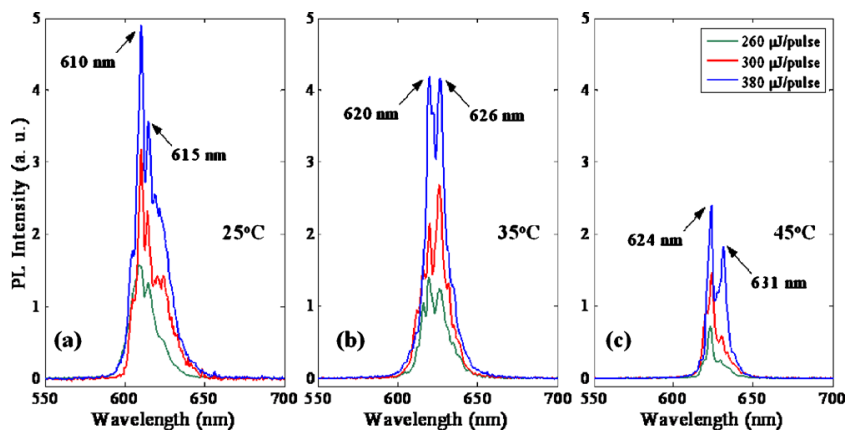

FIG. 2. (Color online) Lasing spectra measured along $\Gamma M$ direction at temperatures of (a) $25^{\circ} \mathrm{C}$, (b) $35^{\circ} \mathrm{C}$, and (c) $45^{\circ} \mathrm{C}$, under different pumping energies of 260,300 , and $380 \mu \mathrm{J} /$ pulse.

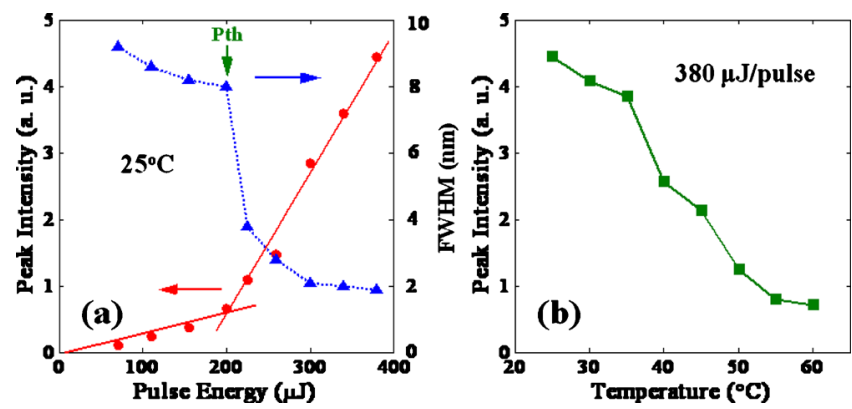

FIG. 3. (Color online) (a) Dependences of peak intensity and FWHM of PL maximum peak on pumping energy at $25^{\circ} \mathrm{C}$. The threshold of pumping energy $\left(P_{\mathrm{th}}\right)$ is around $200 \mu \mathrm{J} /$ pulse. (b) Dependence of lasing peak intensity on temperature. The pumping energy is $380 \mu \mathrm{J} /$ pulse.

temperature is increased, the intensity of lasing peaks is decreased and the wavelength of lasing peaks is red-shifted.

Figure 3(a) shows the dependence of the lasing peak intensity $(610 \mathrm{~nm})$ and the full width at half maximum (FWHM) of the peak as functions of pumping energy at $25^{\circ} \mathrm{C}$. The pumping threshold energy $\left(P_{\mathrm{th}}\right)$ was around $200 \mu \mathrm{J} /$ pulse, above which the peak intensity and FWHM increased and narrowed rapidly, respectively, with the increase in pumping energy. A $1.9 \mathrm{~nm}$ FWHM of lasing peak was obtained at $25^{\circ} \mathrm{C}$, under the pumping energy of $380 \mu \mathrm{J} /$ pulse here. It is worth noticing that, under the same pumping energy of $380 \mu \mathrm{J} /$ pulse, the FWHM of lasing peak at different temperatures, e. g. $620 \mathrm{~nm}$ for $35^{\circ} \mathrm{C}$ and $624 \mathrm{~nm}$ for $45^{\circ} \mathrm{C}$, showed little change compared to that at $25^{\circ} \mathrm{C}$. The dependence of lasing peak intensity on temperature from 25 to $60{ }^{\circ} \mathrm{C}$ is shown in Fig. 3(b), where the pumping energy is fixed at $380 \mu \mathrm{J} /$ pulse. As the temperature increases, the lasing peak intensity decreases, showing a similar behavior to the previous report on lasing from dye-doped H-PDLC transmission grating. ${ }^{15}$ With the increase in temperature, the average effective index of LC will decrease, leading to a reduced index difference of LC droplets and polymer, ${ }^{17}$ thus a weaker light scattering of H-PDLC sample to input light. Therefore, the output light intensity was reduced accordingly.

In general, the H-PDLC 2D PC is slightly anisotropic since the long narrow morphology of the LC droplets tends to align the LCs parallel to the $z$ direction. ${ }^{18}$ Therefore, the index of LC droplets experienced by TE polarization $\left(n_{\mathrm{LC}}\right)$ at temperature of $25^{\circ} \mathrm{C}$ is smaller than the average refractive index of LC $\left[n_{\text {ave }}=\left(2 n_{\mathrm{o}}+n_{\mathrm{e}}\right) / 3\right]$. When the temperature is increased, two mechanisms would influence the refractive index of LC droplets experienced by the TE polarized light. First, the optical anisotropy will be reduced and the TE polarization will experience a higher index of LC droplets, approaching the average refractive index of LC. The actual index change relies on the initial optical anisotropy of the LC droplets (the largest possible change is up to $\Delta n=n_{\text {ave }}-n_{\mathrm{o}}$ $\approx 0.07$ ). Second, the $n_{\text {ave }}$ of LC will be decreased with the increase in temperature but this effect is comparably small $\left(\Delta n_{\text {ave }} \approx-0.01\right.$ from 25 to $\left.45{ }^{\circ} \mathrm{C}\right) .{ }^{17}$ The final LC index experienced by the TE polarization will depend on these two competing mechanisms. As we discussed above, the optical anisotropy reduction should be the dominant factor. In our 

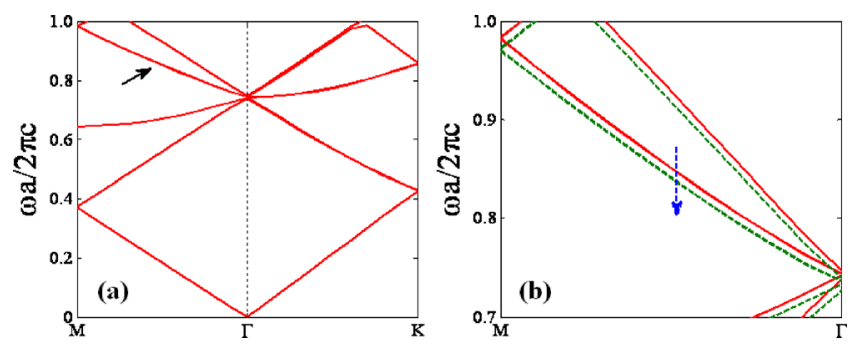

FIG. 4. (Color online) (a) Photonic band structure of the 2D hexagonal H-PDLC PC for TE polarization, where $r=0.30 a, n_{\mathrm{p}}=1.522$, and $n_{\mathrm{LC}}$ $=1.570$. (b) The shift in the band where the laser action occurred with the increase in temperature. Solid line and dashed line represent the band of TE polarization with $n_{\mathrm{LC}}=1.570$ and with $n_{\mathrm{LC}}=1.597$, respectively. The arrows represent the shifting directions with increasing temperature.

experiment, the redshift in lasing spectrum (corresponding to an increased refractive index) with the increase in temperature indicates that indeed the influence from the reduced optical anisotropy is dominant.

The photonic band structure of our 2D hexagonal H-PDLC PC, calculated by the plane wave expansion method, ${ }^{19}$ is shown in Fig. 4(a), assuming $r=0.30 a, n_{\mathrm{p}}$ $=1.522$, and the initial index of LC for TE polarization at the temperature of $25^{\circ} \mathrm{C}, n_{\mathrm{LC}}=1.570$, . The solid red line represents the photonic band of TE polarization and the band where the generated laser is marked by the arrow. When temperature is increased, assuming the experienced index of LC droplets by TE polarization increased from $n_{\mathrm{LC}}=1.570$ to 1.597 and the index of polymer is unchanged, the photonic band of TE polarization shifted downward, as shown in Fig. 4(b) by the green dash line. The downward shift in photonic band, shown by the arrow, leads to a decrease in the lasing frequency, therefore, an increase in wavelength. Therefore, the redshift in the experimental lasing spectra with increased temperature (in Fig. 2), is quite consistent with our theoretical analysis discussed above.

\section{CONCLUSIONS}

In conclusion, we demonstrated the temperature tunable properties of lasing from a dye-doped 2D H-PDLC PC with hexagonal lattice along $\Gamma \mathrm{M}$ direction. When the temperature increased from 25 to $45{ }^{\circ} \mathrm{C}$, a redshift in lasing peaks has been observed. The decrease in LC droplets' anisotropy with the increased temperature for TE polarization is the major underlying reason for downward movement of photonic band, which eventually leads to the redshift in lasing peaks.

${ }^{1}$ T. J. Bunning, L. V. Natarajan, V. P. Tondiglia, and R. L. Sutherland, Annu. Rev. Mater. Sci. 30, 83 (2000).

${ }^{2}$ M. J. Escuti, J. Qi, and G. P. Crawford, Appl. Phys. Lett. 83, 1331 (2003).

${ }^{3}$ Y. J. Liu and X. W. Sun, Jpn. J. Appl. Phys., Part 1 46, 6634 (2007).

${ }^{4}$ G. S. He, T.-C. Lin, V. K. S. Hsiao, A. N. Cartwright, P. N. Prasad, L. V. Natarajan, V. P. Tondiglia, R. Jakubiak, R. A. Vaia, and T. J. Bunning, Appl. Phys. Lett. 83, 2733 (2003).

${ }^{5}$ R. Jakubiak, L. V. Natarajan, V. Tondiglia, G. S. He, P. N. Prasad, T. J. Bunning, and R. A. Vaia, Appl. Phys. Lett. 85, 6095 (2004).

${ }^{6}$ Y. J. Liu, X. W. Sun, P. Shum, H. P. Li, J. Mi, W. Ji, and X. H. Zhang, Appl. Phys. Lett. 88, 061107 (2006).

${ }^{7}$ H. Kogelnik and C. V. Shank, J. Appl. Phys. 43, 2327 (1972).

${ }^{8}$ R. Jakubiak, V. P. Tondiglia, L. V. Natarajan, R. L. Sutherland, P. Lloyd, T. J. Bunning, and R. A. Vaia, Adv. Mater. 17, 2807 (2005).

${ }^{9}$ D. Luo, X. W. Sun, H. T. Dai, Y. J. Liu, H. Z. Yang, and W. Ji, Appl. Phys. Lett. 95, 151115 (2009).

${ }^{10}$ K. Sakoda, Opt. Express 4, 167 (1999).

${ }^{11}$ D. E. Lucchetta, L. Criante, O. Francescangeli, and F. Simoni, Appl. Phys. Lett. 84, 837 (2004).

${ }^{12}$ D. E. Lucchetta, L. Criante, O. Francescangeli, and F. Simoni, Mol. Cryst. Liq. Cryst. 441, 97 (2005).

${ }^{13}$ V. K. S. Hsiao, C. Lu, G. S. He, M. Pan, A. N. Cartwright, P. N. Prasad, R. Jakubiak, R. A. Vaia, and T. J. Bunning, Opt. Express 13, 3787 (2005).

${ }^{14}$ H. Harada, M. Nakatsu, and H. Naito, Jpn. J. Appl. Phys., Part 2 44, L915 (2005).

${ }^{15}$ Y. J. Liu, X. W. Sun, H. I. Elim, and W. Ji, Appl. Phys. Lett. 90, 011109 (2007).

${ }^{16}$ M. B. Christiansen, A. Kristensen, S. Xiao, and N. A. Mortensen, Appl. Phys. Lett. 93, 231101 (2008).

${ }^{17}$ T. T. Alkeskjold, J. Lægsgaard, A. Bjarklev, D. S. Hermann, A. Anawati, J. Broeng, J. Li, and S.-T. Wu, Opt. Express 12, 5857 (2004).

${ }^{18}$ M. S. Li, S. T. Wu, and A. Y.-G. Fuh, Appl. Phys. Lett. 88, 091109 (2006).

${ }^{19}$ M. Notomi, Phys. Rev. B 62, 10696 (2000). 